\title{
Identification of $\mathrm{CHCHD2}$ mutations in patients with Alzheimer's disease, amyotrophic lateral sclerosis and frontotemporal dementia in China
}

\author{
XIXI LIU $^{1}$, BIN JIAO ${ }^{1-3}$, WEIWEI ZHANG ${ }^{1}$, TINGTING XIAO ${ }^{1}$,

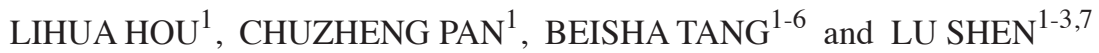

\begin{abstract}
${ }^{1}$ Department of Neurology, Xiangya Hospital; ${ }^{2}$ Key Laboratory of Hunan Province in Neurodegenerative Disorders, Central South University, Changsha, Hunan 410008; ${ }^{3}$ National Clinical Research Center for Geriatric Diseases, Changsha, Hunan 410078; ${ }^{4}$ Parkinson's Disease Center of Beijing Institute for Brain Disorders, Beijing 100069;

${ }^{5}$ Collaborative Innovation Center for Brain Science, Shanghai 200032; ${ }^{6}$ Collaborative Innovation Center for Genetics and Development, Shanghai 200433; ${ }^{7}$ Key Laboratory of Organ Injury,

Aging and Regenerative Medicine of Hunan, Changsha, Hunan 410008, P.R. China
\end{abstract}

Received December 1, 2017; Accepted April 26, 2018

DOI: $10.3892 / \mathrm{mmr} .2018 .8962$

\begin{abstract}
Recently, the coiled-coil-helix-coiled-coil-helix domain 2 ( $\mathrm{CHCHD}$ ) gene was identified as a possible causative gene for Parkinson's disease (PD). Three other neurodegenerative diseases, Alzheimer's disease (AD), amyotrophic lateral sclerosis (ALS) and frontotemporal dementia (FTD), share significant overlaps with PD in clinical phenotypes, pathological features and genetic heredities, and it is still unclear whether $C H C H D 2$ variants could explain these three diseases. The present study screened all exons of the $\mathrm{CHCHD} 2$ gene in a total of 780 patients (511 AD, 181 ALS and 88 FTD) and 500 healthy controls from the Chinese Han population. Two missense variants, 5C $>$ T (Pro2Leu) and 238A $>$ G (Ile80Val), were identified in five unrelated patients with AD while no mutations were observed in patients with ALS or FTD. These mutations have been reported as low-frequency variants in the Ex AC database with frequencies of 0.0075 and 0.000025 . Pro2 Leu, however, was also detected in controls and was confirmed to have no significant association with the risk for AD; Ile80Val was not detected in any normal controls, suggesting that the CHCHD2 gene may be associated with AD in the Chinese Han population.
\end{abstract}

\section{Introduction}

The coiled-coil-helix-coiled-coil-helix domain (CHCHD)containing proteins are small mitochondrial proteins with

Correspondence to: Dr Lu Shen, Department of Neurology, Xiangya Hospital, Central South University, 87 Xiangya Road, Changsha, Hunan 410008, P.R. China

E-mail: shenlu2505@126.com

Key words: Alzheimer's disease, amyotrophic lateral sclerosis, frontotemporal dementia, coiled-coil-helix-coiled-coil-helix domain 2 important functions. Mutations of CHCHD genes have been identified to be associated with various human neurodegenerative diseases (1). CHCHD10, which is a CHCHD protein, was identified to be associated with amyotrophic lateral sclerosis (ALS), frontotemporal dementia (FTD) and Alzheimer's disease (AD) in Chinese population $(2,3)$. Recently, the CHCHD2 gene was identified as a possible causative gene for Parkinson's disease (PD). A missense mutation (Thr61Ile) in this gene was first detected in a multigenerational Japanese family with autosomal dominant PD (ADPD) (4). Several subsequent efforts have been made to confirm the association between the CHCHD2 gene and PD in other ethnicities, including European and Chinese populations. Jansen et al (5) reported three novel putative pathogenic variants (Ala32Thr, Pro34Leu, and Ile80Val) in patients with PD from a western European population; another study identified a heterozygous variant (182C $>\mathrm{T}$; Thr61Ile) in an ADPD pedigree in a Chinese population (6). The CHCHD2 gene is located on chromosome 7p11.2 and contains four exons encoding 151 amino acids with a predicted $\mathrm{N}$-terminal mitochondrial targeting sequence (7). The CHCHD2 protein is a small mitochondrial protein that serves as one of the negative regulators of mitochondria-mediated apoptosis. The knockdown of CHCHD2 promoted a significant increase in nuclear fragmentation and in phosphatidylserine exposure, both of which are hallmarks of apoptosis (8).

Neurodegenerative disorders are conditions that have yet to be fully elucidated. Nevertheless, different types of neurodegenerative disorders are closely related, including the four major types: PD, AD, ALS and FTD. Although they are different diseases with distinct features, they manifest overlapping clinical phenotypes, pathologic features and genetic backgrounds. For instance, the PD-related genetic variant rs76904798 of leucine-rich repeat kinase 2 (LRRK2) is found to be a common genetic risk variant for late-onset AD (LOAD) susceptibility in a northern Han Chinese people (9). Expansions in the C9orf72 gene are most frequently associated 
with ALS-FTD and can be combined with symmetrical Parkinsonism; FTD in patients with mutations in the gene that encodes microtubule-associated protein tau (MAPT) can also manifest as symmetrical Parkinsonism (10).

In addition, mitochondrial dysfunction has been described in neurodegenerative disorders. In addition to CHCHD2, the proteins that are associated with familial PD-PTEN-induced putative kinase 1 (PINK1), DJ-1, alpha-synuclein and LRRK2-are either mitochondrial proteins or associated with mitochondria, and all interface with the pathways of oxidative stress and free radical damage (11). In $\mathrm{AD}$, aggregation of $\beta$-amyloid $(A \beta)$ is central to initiating AD pathogenesis. In the brain and in isolated mitochondria, exposure to $A \beta$ inhibits key mitochondrial enzymes (12). As mentioned above, mutations of $\mathrm{CHCHD} 10$ are associated with ALS and/or FTD, AD.

In this study, to confirm the potential role of $C H C H D 2$ in these three diseases, we assessed the prevalence of $\mathrm{CHCHD} 2$ mutations in AD, ALS and FTD patients.

\section{Patients and methods}

Patients. This study recruited a total of 511 AD patients (436 sporadic AD and 75 probands from FAD families, mean age at onset was $66.2 \pm 5.5$ years, male: $44.0 \%), 181$ ALS patients (166 sporadic ALS and 15 familial ALS, mean age at onset was $48.1 \pm 13.4$ years, male: $67.9 \%$ ) and 88 FTD patients (77 sporadic FTD and 11 familial FTD, mean age at onset was $53.3 \pm 9.7$ years, male: $42.0 \%$ ) from mainland China in 2014 to 2016. The diagnoses of probable or possible AD according to the NINCDS-ADRDA criteria were made by 2 or more experienced neurologists in Xiangya Hospital. The diagnoses of ALS were made according to the El Escorial revised criteria. The diagnoses of FTD met the Lund-Manchester criteria (13-15). Neuroradiological examinations for example MRI, was assessed with patients diagnosed as probable or possible AD and FTD in this study. We have excluded $\mathrm{AD}, \mathrm{FTD}$ and ALS patients carrying disease-causing gene like PSEN1, PSEN2, APP, MAPT, GRN, C9orf72, TREM2, CHCHD10, SODI, TARDBP, FUS (3,16-20). Additionally, the $A P O E$ genotype was available for all AD patients. A total of 500 healthy unrelated age-matched Chinese individuals without a history of neurodegenerative disease were recruited from the Xiangya Wellness Center as a control group. Written informed consent for participation in the study was obtained from all subjects. For patients who can understand our study, we asked for the consent of the patients, and for severe patients, we sought the consent of the patient's guardian or immediate family members. This study was conducted in accordance with the Declaration of Helsinki and was approved by the Expert Committee of Xiangya Hospital of Central South University in China.

DNA isolation and genotyping methods. Genomic DNA was extracted from peripheral blood leukocytes from all patients and controls. The quality and quantity of DNA were assessed with a fluorometer. All DNA samples were diluted to $50 \mathrm{ng} / \mathrm{ml}$. Polymerase chain reaction (PCR) was performed on the exonic regions of $C H C H D 2$. The $C H C H D 2$ sequencing (exons 1-4) was amplified using primers designed according to the GenBank entries (data not shown). Each PCR product was sequenced using forward and reverse primers identical to the ones used in PCR with BigDye terminator v3.1 sequencing chemistry on an ABI 3730x1 DNA analyzer (Applied Biosystems; Thermo Fisher Scientific, Inc., Waltham, MA, USA). The DNA sequences were analyzed using Sequencher software.

Statistical analysis. When a mutation was detected, we first confirmed whether it was a novel or rare $(\mathrm{MAF}<1 \%)$ mutation by comparison with sequences in the ExAC (exac. broadinstitute.org/) and 1000 Genomes Project databases (www.1000genomes.org/) and with direct sequencing of healthy controls. We then use bioinformatics prediction tools like Mutation Taster (mutationtaster.org), REVEL (sites. google.com/site/revelgenomics/downloads), CADD (cadd. gs.washington.edu/download) to predict the pathogenicity of the mutation. Statistical analysis of clinical data was performed using IBM SPSS 19.0 (IBM Corp., Armonk, NY, USA). To compare the differences among the patients and controls, the Fisher's exact test was used. The threshold of statistical significance was set at $\mathrm{P}<0.05$. Descriptive statistics were expressed as the mean \pm the standard deviation.

\section{Results}

The demographic features of 511 AD, 181 ALS and 88 FTD cases and 500 controls are shown in Table I. The Clinical features and $A P O E$ genotype of the carriers is presented in Table II.

Two rare heterozygous variants of CHCHD2, $5 \mathrm{C}>\mathrm{T}$ (Pro2Leu) and $238 \mathrm{~A}>G$ (Ile80Val), were found in five of $511 \mathrm{AD}$ patients. The variant Pro2Leu was identified in four patients with typical symptoms of cognitive impairment; three of them had sporadic $\mathrm{AD}$, and one had a family history of AD. The patient with a family history developed progressive memory impairment at the age of 67. At the onset, she easily forgot what she had just done. Then, she began to forget her relatives' names and could not find her way home. Finally, multiple cognitive domains were impaired. Her Mini-Mental State Examination (MMSE) score was 5 of 30 points; her Montreal cognitive assessment scale (MoCA) score was 4 of 30 points. Her mother also had symptoms of memory loss, according to the recollection of the patient's family. One of the proband's sisters (Fig. 1A, M6937: II3) who had similar symptoms also carried the same variant. Unfortunately, DNA samples from other family members were unavailable for genetic analysis. This variant showed a frequency of 0.007475 in the ExAC database. We also detected this variant with equal frequency in our control group (Table III), which indicated that there was no significant association between Pro2Leu and the risk for AD in our cohort.

The variant Ile80Val was identified in a male patient who developed progressive memory loss at the age of 67 . A year later, he had a change of personality. Specifically, he became less talkative and more aggressive than he used to be. The patient went to see a doctor at the age of 70; his MMSE score was 5 of 30 points, and his MoCA score was 0 of 30 points. His mother began suffering from memory loss at the age of 81 ; two years later, she lost the ability to live independently and died because of pulmonary infection. One of the proband's younger sister (Fig. 1A, M31801: II-3) and his two sons (III-1 and III-2) 
Table I. Demographic information of patients and control groups.

\begin{tabular}{lcccc}
\hline Variable & AD & ALS & FTD & Control \\
\hline Cases, $\mathrm{n}$ & 511 & 181 & 88 & 500 \\
No. of male cases, $\mathrm{n}(\%)$ & $225(44.0)$ & $123(68.0)$ & $37(42.0)$ & $253(50.6)$ \\
Age at onset, years & $66.2 \pm 5.5$ & $48.1 \pm 13.4$ & $53.3 \pm 9.7$ & - \\
Age at examination, years & $70.0 \pm 5.7$ & $50.8 \pm 12.3$ & $58.5 \pm 11.5$ & $69.3 \pm 6.1$ \\
MMSE score & $18.13 \pm 7.78$ & - & $16.31 \pm 9.45$ & $28.7 \pm 1.4$
\end{tabular}

Data are presented as the mean \pm standard deviation. MMSE, Mini-Mental State Examination; AD, Alzheimer's disease; ALS, amyotrophic lateral sclerosis; FTD, frontotemporal dementia; -, data not available.

Table II. Clinical features of Alzheimer's disease patients carrying variants of the $C H C H D 2$ gene.

\begin{tabular}{|c|c|c|c|c|c|}
\hline Characteristic & M6937 & M14200 & M14851 & M24736 & M31801 \\
\hline Sex & Female & Female & Female & Female & Male \\
\hline Family history & $\mathrm{Y}$ & $\mathrm{N}$ & $\mathrm{N}$ & $\mathrm{N}$ & $\mathrm{Y}$ \\
\hline Age at onset (years) & 67 & 60 & 82 & 53 & 67 \\
\hline Age at examination (years) & 79 & 64 & 91 & 58 & 70 \\
\hline MMSE & 5 & 1 & 4 & 22 & 5 \\
\hline $\mathrm{MoCA}$ & 4 & 1 & 6 & 9 & 0 \\
\hline Variant & 5C $>\mathrm{T}$ (Pro2Leu) & 5C $>\mathrm{T}($ Pro2Leu $)$ & $5 \mathrm{C}>\mathrm{T}($ Pro2Leu $)$ & 5C $>\mathrm{T}$ (Pro2Leu) & $238 \mathrm{~A}>\mathrm{G}(\mathrm{Ile} 80 \mathrm{Val})$ \\
\hline APOE allele & $\varepsilon 4 / \varepsilon 2$ & $\varepsilon 3 / \varepsilon 3$ & $\varepsilon 3 / \varepsilon 3$ & $\varepsilon 3 / \varepsilon 3$ & $\varepsilon 3 / \varepsilon 3$ \\
\hline
\end{tabular}

CHCHD2, coiled-coil-helix-coiled-coil-helix domain 2; Y, yes; N, no; MMSE, Mini-Mental State Examination; MoCA, Montreal cognitive assessment scale; APOE, apolipoprotein E.

agreed to participate in our study. We performed cognitive evaluation and genetic analysis of these three members, it turned out that one of his son (III-2) carried this variant while others did not (all of them are cognitively normal). We will continue to monitor whether he develops any symptoms of AD. This variant showed a frequency of 0.000025 in ExAC and was not detected in data from the 1000 Genomes Project and was also absent in our 500 healthy control individuals. The variant sequences in patients and references are presented in Fig. 1B.

Both of these two variants were highly conserved among the primary species (Fig. 1C) and were predicted to be detrimental based on the Mutation Taster. The variant $5 \mathrm{C}>\mathrm{T}$ (Pro2Leu) was predicted to be the $1 \%$ most deleterious and the variant $238 \mathrm{~A}>\mathrm{G}$ (Ile80Val) to be the $10 \%$ most deleterious according to CADD. However, these two variants are less likely to cause disease in REVEL prediction (Table III).

No CHCHD2 variant was detected in patients with ALS or FTD. In our 181 ALS and 88 FTD samples, Sanger sequencing ruled out coding mutations in $C H C H D 2$, suggesting that CHCHD2 might not be a risk gene in these two diseases.

\section{Discussion}

CHCHD2 co-expresses with other genes of the oxidative phosphorylation pathway, and the CHCHD2 protein serves as a transcription factor to activate the oxygen response element (ORE) in the COX4I2 gene (21). Funayama et al (4) first identified a missense mutation (Thr61Ile) of $\mathrm{CHCHD2}$ in a multigenerational Japanese family with ADPD. Subsequently, they found two more mutations, one missense mutation (434G>A, Arg145Gln) and one splice-site mutation $(300+5 \mathrm{G}>\mathrm{A})$, in two other families. And none of these three mutations was noted in the 559 unaffected Japanese controls (4). Of these mutations, only the Thr61Ile mutation was confirmed to cosegregate in two independent families with ADPD (22). In addition to PD, recent studies have reported that $C H C H D 2$ expression was increased in neural stem cell lines derived from a patient with Huntington's disease, and gene variants of $\mathrm{CHCHD} 2$ were also detected in patients with Lewy body disease (LBD) $(23,24)$ Based on these observations, the $C H C H D 2$ gene might be involved in various neurodegenerative diseases.

In this study, we detected two single nucleotide variants of CHCHD2 (Pro2 Leu and Ile80 Val) in five AD patients. All of these five patients had typical symptoms of AD. Pro2Leu was confirmed to have different frequencies in patients with sporadic PD and controls by Funayama et al (4) and Shi et al (6). Another similar research that may be carried out at the same time with this study found four variants of CHCHD2 gene in AD and FTD patients and one of them is Pro2Leu (25). However, in terms of our results, this variant might not be significantly associated with AD. Ile80Val 
Table III. Frequencies and in silico analyses of $C H C H D 2$ variants.

\begin{tabular}{|c|c|c|c|c|c|c|c|}
\hline \multirow[b]{2}{*}{ Variants } & \multicolumn{3}{|c|}{ Minor allele frequency } & \multirow[b]{2}{*}{ Mutation taster } & \multirow[b]{2}{*}{ REVEL score ${ }^{\mathrm{b}}$} & \multicolumn{2}{|c|}{ CADD } \\
\hline & $\mathrm{AD}$ & Control & $\mathrm{P}$-value & & & Raw score & PHRED $^{c}$ \\
\hline 5C>T(Pro2Leu) & 0.0078 & 0.006 & $1^{\mathrm{a}}$ & Disease causing & 0.275 & 4.554172 & 24.4 \\
\hline 238A $>\mathrm{G}($ Ile80Val $)$ & 0.0020 & - & - & Disease causing & 0.009 & 1.187786 & 11.68 \\
\hline
\end{tabular}

${ }^{a}$ Fisher's exact test; ${ }^{b}$ The threshold value set in the present study was 0.4 , above which a variant would be classified as pathogenic; ${ }^{\text {PHRED-like }}$ scaled C-score. A scaled C-score of $\geq 10$ indicates that these are predicted to be the $10 \%$ most deleterious substitutions that you can do to the human genome, a score of $\geq 20$ indicates the $1 \%$ most deleterious. CHCHD2, coiled-coil-helix-coiled-coil-helix domain 2; AD, Alzheimer's disease; REVEL, Rare Exome Variant Ensemble Learner; CADD, Combined Annotation Dependent Depletion; PHRED, Phil's Read Editor.
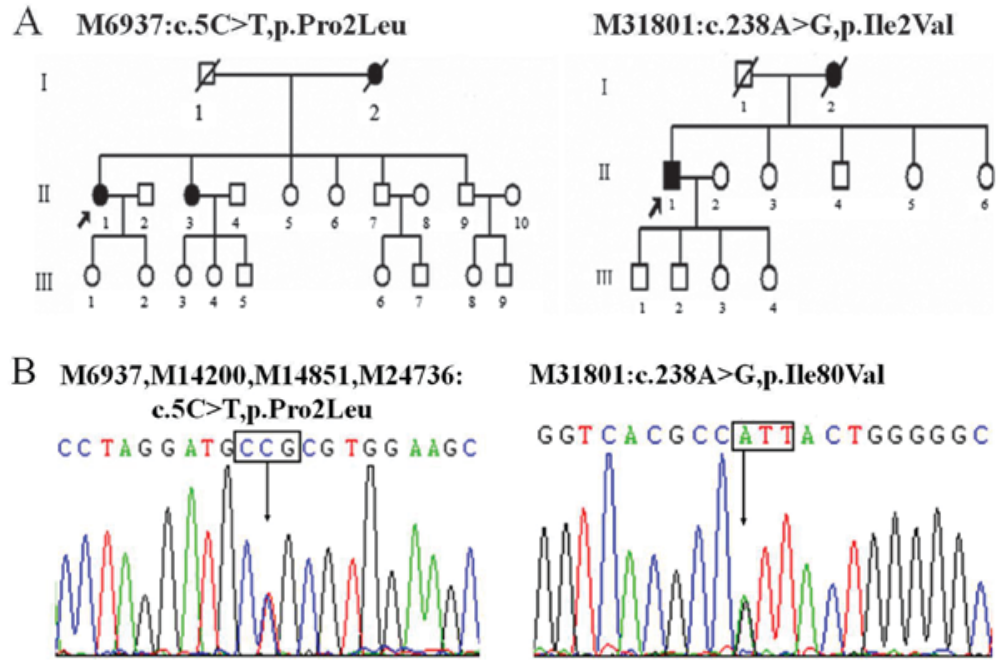

Control:

C CTAG A AT GCCGTG TGGA A GC

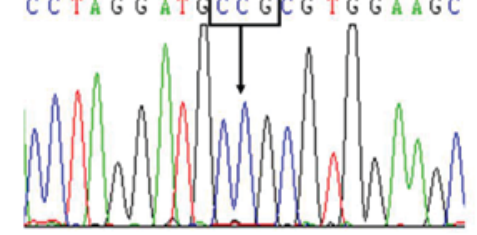

C 5C $>$ T $($ Pro2Leu $)$

Patient $\quad L$

Danio PFGSRSRTSRISPPSYSSPAPUARAPPRSY

Gallus IPEGGISRSRVYAPPASRAPQ--MRAAPPAPT

Xenopus IDFGSESRTSRYAPPASRAPh--IIRPAPPPA

Mus HEGGSSRTSRVIPPASRAPQ--RRAPRRAP

Rattus IPEGSESRTSRVIPPASRAPQ--IIRALPRRAP.

Canis HPGGSSRASBMAPPASRRPQ--MRRAPRPAP

Bos IPGGSRSTSRYAPPASRAPQ--IIRUAPRPAP

Homo HERGSRSRTSBMPPASRRPQ--IIRAPPRPAP

Pan $\quad$ FEGSESRTSEMAPPASRAPQ-NIIRAPRPAP

Macaca IERGSRSRTSBMAPPASRAPQ--MRGRPRPAP

\section{M31801:c.238A >G,p.Ile80Val}

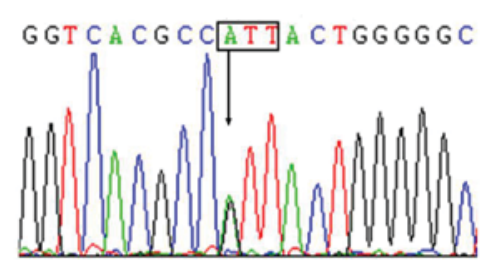

Control:

G GT CACGCCATTCTGGGGGC

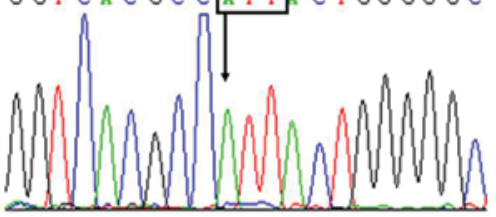

238A $>$ G (lle80Val)

\begin{tabular}{|c|c|}
\hline atient & $y$ \\
\hline Danio & AQMASTAGYAVGSAVGHIIGHUITGGPGGGH \\
\hline allus & AQHATTAGGVAVGSAVGHTIGHAEFGGPGGGSS \\
\hline enop & AQMATTMGVAVGSAVGHIGK \\
\hline & AQMKTTAAC \\
\hline Rattus & AQHATT \\
\hline 15 & AOMATTA \\
\hline cos & AQHATTAAGVAVGSAVGHTLGHATIGGFSGGSS \\
\hline Iomo & 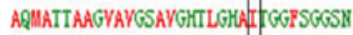 \\
\hline an & AQHATTARGVAVGSAVGTILGHAITGGPSGGSN \\
\hline & AQMATTAGVVAVGSAVGHTLGHATIGGPSGGSN \\
\hline
\end{tabular}

Figure 1. (A) Pedigrees of families with Alzheimer's disease carrying the CHCHD2 variant; the proband is indicated by an arrow. (B) Sanger sequencing revealed two CHCHD2 variants in 5 patients and controls; M6937, M14200, M14851, M24736: c.5C>T, Pro2Leu; and M31801: c.238G>A, Ile2Val. (C) Cross-species protein conservation of $\mathrm{CHCHD} 2, \mathrm{c} .5 \mathrm{C}>\mathrm{T}$ and c.238G $>\mathrm{A}$ were highly conserved residues across species. CHCHD2, coiled-coil-helix-coiled-coil-helix domain 2.

was first identified in a PD patient in a western European population (5). According to the ExAC database and the 1000 Genomes Project, this variant has not been previously detected in an East Asian population. Ile80Val is located in a transmembrane domain and was predicted to be detrimental based on the Mutation Taster and CADD (8). Nonetheless, the
REVEL predicted a score of 0.009 for this mutation, which might not cause disease. Considering the reasons given above, CHCHD2 is not likely to be a causative gene of AD in the Chinese population but might be associated with $\mathrm{AD}$ and AD might share a common pathway with PD in mitochondrial dysfunction. However, the pathogenicity of this variant 
remains uncertain. Further functional experiments are needed to investigate how $\mathrm{CHCHD} 2$ plays a role in $\mathrm{AD}$ and $\mathrm{PD}$.

No mutation of $C H C H D 2$ was observed in either the 181 ALS or the 88 FTD patients. There are several possible explanations for these negative results. The first possibility is that $\mathrm{CHCHD} 2$ might not be associated with these two diseases in the Chinese population, although many pathogenic gene mutations can be detected in ALS, FTD and PD. Another explanation for these results is that the $C H C H D 2$ gene might have genetic heterogeneity in different ethnic groups and since some $C H C H D 2$ gene mutations are very rare, more samples should be included to provide more solid evidence.

Rare coding variants play major roles in disease causation and might contribute to the missing heritability from genome-wide association studies. In this study, to find out whether these mutations play a role in the disease, we use bioinformatics prediction tools to predict the pathogenicity of the mutation and compared its frequency with healthy control. However, the present study has several limitations. Firstly, association study requires a larger sample size to detect rare variants with modest effect sizes with high statistical power. Secondly, bioinformatics prediction tools rely on pathogenicity assertions from existing databases, which might be inaccurate and incomplete.

In general, this study identified a novel mutation of the CHCHD2 gene in Chinese AD patients, while no mutation of $C H C H D 2$ was observed in either ALS or FTD patients, suggesting that the $C H C H D 2$ gene might be associated with $\mathrm{AD}$ in the Chinese Han population. Further screening should be conducted in a large number of samples and in different ethnicities and further functional experiments are needed.

\section{Acknowledgements}

Not applicable.

\section{Funding}

The present study was supported through the National Natural Science Foundation of China (grant nos. 81671075 and 81701134).

\section{Availability of data and materials}

The datasets used and/or analyzed during the current study are available from the corresponding author on reasonable request.

\section{Authors' contributions}

XL, BJ, LS and BT designed the study. XL, WZ, TX, LH, CP and $\mathrm{BT}$ conducted the experiments, and analyzed and interpreted the data. XL and LS wrote the manuscript. BT and LS supervised the study. BJ and LS provided financial support.

\section{Ethics approval and consent to participate}

The present study was conducted in accordance with the Declaration of Helsinki and was approved by the Expert Committee of Xiangya Hospital of Central South University in China (Hunan, China; ref. no. 201603198). Written informed consent for participation in the study was obtained from all subjects; consent was obtained from the patient's guardian or immediate family member for those without the capacity to consent.

\section{Consent for publication}

Written informed consent was obtained from all subjects; consent was obtained from the patient's guardian or immediate family member for those without the capacity to consent.

\section{Competing interests}

The authors declare that they have no competing interests.

\section{References}

1. Zhou ZD, Saw WT and Tan EK: Mitochondrial CHCHD-containing proteins: Physiologic functions and link with neurodegenerative diseases. Mol Neurobiol 54: 5534-5546, 2017.

2. Bannwarth S, Ait-El-Mkadem S, Chaussenot A, Genin EC, Lacas-Gervais S, Fragaki K, Berg-Alonso L, Kageyama Y, Serre V, Moore DG, et al: A mitochondrial origin for frontotemporal dementia and amyotrophic lateral sclerosis through CHCHD10 involvement. Brain 137: 2329-2345, 2014.

3. Xiao T, Jiao B, Zhang W, Pan C, Wei J, Liu X, Zhou Y, Zhou L, Tang B and Shen L: Identification of CHCHD10 mutation in chinese patients with Alzheimer disease. Mol Neurobiol 54: 5243-5247, 2017.

4. Funayama M, Ohe K, Amo T, Furuya N, Yamaguchi J, Saiki S, Li Y, Ogaki K, Ando M, Yoshino H, et al: CHCHD2 mutations in autosomal dominant late-onset Parkinson's disease: A genome-wide linkage and sequencing study. Lancet Neurol 14: 274-282, 2015.

5. Jansen IE, Bras JM, Lesage S, Schulte C, Gibbs JR, Nalls MA Brice A, Wood NW, Morris H, Hardy JA, et al: CHCHD2 and Parkinson's disease. Lancet Neurol 14: 678-679, 2015.

6. Shi CH, Mao CY, Zhang SY, Yang J, Song B, Wu P, Zuo CT, Liu YT, Ji Y, Yang ZH, et al: CHCHD2 gene mutations in familial and sporadic Parkinson's disease. Neurobiol Aging 38: 217.e9-e217.e13, 2016.

7. Puschmann A, Dickson DW, Englund E, Wszolek ZK and Ross OA: CHCHD2 and Parkinson's disease. Lancet Neurol 14 679, 2015.

8. Liu Y, Clegg HV, Leslie PL, Di J, Tollini LA, He Y, Kim TH, Jin A, Graves LM, Zheng J and Zhang Y: CHCHD2 inhibits apoptosis by interacting with $\mathrm{Bcl}-\mathrm{x} \mathrm{L}$ to regulate $\mathrm{Bax}$ activation. Cell Death Differ 22: 1035-1046, 2015.

9. Zhu XC, Cao L, Tan MS, Jiang T, Wang HF, Lu H, Tan CC, Zhang W, Tan L and Yu JT: Association of Parkinson's Disease GWAS-linked loci with Alzheimer's disease in Han Chinese. Mol Neurobiol 54: 308-318, 2017.

10. Baizabal-Carvallo JF and Jankovic J: Parkinsonism, movement disorders and genetics in frontotemporal dementia. Nat Rev Neurol 12: 175-185, 2016.

11. Schapira AH: Mitochondria in the aetiology and pathogenesis of Parkinson's disease. Lancet Neurol 7: 97-109, 2008.

12. Querfurth HW and LaFerla FM: Alzheimer's disease. N Engl J Med 362: 329-344, 2010.

13. McKhann G, Drachman D, Folstein M, Katzman R, Price D and Stadlan EM: Clinical diagnosis of Alzheimer's disease: Report of the NINCDS-ADRDA Work Group under the auspices of Department of Health and Human Services Task Force on Alzheimer's Disease. Neurology 34: 939-944, 1984.

14. Brooks BR: El Escorial World Federation of Neurology criteria for the diagnosis of amyotrophic lateral sclerosis. Subcommittee on Motor Neuron Diseases/Amyotrophic Lateral Sclerosis of the World Federation of Neurology Research Group on Neuromuscular Diseases and the El Escorial 'Clinical limits of amyotrophic lateral sclerosis' workshop contributors. J Neurol Sci 124 (Suppl): S96-S107, 1994. 
15. Clinical and neuropathological criteria for frontotemporal dementia. The Lund and Manchester Groups. J Neurol Neurosurg Psychiatry 57: 416-418, 1994

16. Tang M, Gu X, Wei J, Jiao B, Zhou L, Zhou Y, Weng L, Yan X, Tang B, Xu J and Shen L: Analyses MAPT, GRN, and C9orf72 mutations in Chinese patients with frontotemporal dementia. Neurobiol Aging 46: 235.e11-e15, 2016.

17. Jiao B, Xiao T, Hou L, Gu X, Zhou Y, Zhou L, Tang B, Xu J and Shen L: High prevalence of CHCHD10 mutation in patients with frontotemporal dementia from China. Brain 139: e21, 2016.

18. Jiao B, Liu X, Tang B, Hou L, Zhou L, Zhang F, Zhou Y, Guo J, Yan X and Shen L: Investigation of TREM2, PLD3, and UNC5C variants in patients with Alzheimer's disease from mainland China. Neurobiol Aging 35: 2422.e9-e2422. e11, 2014.

19. Jiao B, Tang B, Liu X, Xu J, Wang Y, Zhou L, Zhang F, Yan X, Zhou Y and Shen L: Mutational analysis in early-onset familial Alzheimer's disease in Mainland China. Neurobiol Aging 35: 1957.e1-e6, 2014.

20. Hou L, Jiao B, Xiao T, Zhou L, Zhou Z, Du J, Yan X, Wang J, Tang B and Shen L: Screening of SOD1, FUS and TARDBP genes in patients with amyotrophic lateral sclerosis in central-southern China. Sci Rep 6: 32478, 2016.
21. Aras S, Pak O, Sommer N, Finley R Jr, Hüttemann M, Weissmann $\mathrm{N}$ and Grossman LI: Oxygen-dependent expression of cytochrome c oxidase subunit 4-2 gene expression is mediated by transcription factors RBPJ, CXXC5 and CHCHD2. Nucleic Acids Res 41: 2255-2266, 2013.

22. Funayama M and Hattori N: CHCHD2 and Parkinson's disease-authors' reply. Lancet Neurol 14: 682-683, 2015.

23. Ogaki K, Koga S, Heckman MG, Fiesel FC, Ando M, Labbé C, Lorenzo-Betancor O, Moussaud-Lamodière EL, Soto-Ortolaza AI, Walton RL, et al: Mitochondrial targeting sequence variants of the CHCHD2 gene are a risk for Lewy body disorders. Neurology 85: 2016-2025, 2015.

24. Feyeux M, Bourgois-Rocha F, Redfern A, Giles P Lefort N, Aubert S, Bonnefond C, Bugi A, Ruiz M, Deglon N, et al: Early transcriptional changes linked to naturally occurring Huntington's disease mutations in neural derivatives of human embryonic stem cells. Hum Mol Genet 21: 3883-3895, 2012.

25. Che XQ, Zhao QH, Huang Y, Li X, Ren RJ, Chen SD, Guo QH and Wang G: Mutation screening of the CHCHD2 gene for Alzheimer's Disease and Frontotemporal Dementia in Chinese Mainland Population. J Alzheimers Dis 61: 1283-1288, 2018. 